\title{
Evolving optimal swimming in different fluids: a study inspired by batoid fishes
}

\author{
V. Cacucciolo *, F. Corucci *, M. Cianchetti and C. Laschi \\ The BioRobotics Institute, Scuola Superiore Sant'Anna, Pisa, Italy \\ \{v.cacucciolo, f.corucci, m.cianchetti, c.laschi $\}$ Osssup.it
}

\begin{abstract}
For their efficient and elegant locomotion, batoid fishes (e.g. the manta ray) have been widely studied in biology, and also taken as a source of inspiration by engineers and roboticists willing to replicate their propulsion mechanism in order to build efficient swimming machines. In this work, a new model of an under-actuated compliant wing is proposed, exhibiting both the oscillatory and undulatory behaviors underlying $b a$ toid propulsion mechanism. The proposed model allowed an investigation of the co-evolution of morphology and control, exploiting dynamics emergent from the interaction between the environment and the mechanical properties of the soft materials. Having condensed such aspects in a mathematical model, we studied the adaptability of a batoid-like morphology to different environments. As for biology, our main contribution is an exploration of the parameters linking swimming mechanics, morphology and environment. This can contribute to a deeper understanding of the factors that led various species of the batoid group to phylogenetically adapt to different environments. From a robotics standpoint, this work offers an additional example remarking the importance of morphological computation and embodied intelligence. A direct application can be an under-water soft robot capable of adapting morphology and control to reach the maximum swimming efficiency.
\end{abstract}

Keywords: Bio-mimetics, embodied intelligence, evolutionary robotics, genetic algorithms.

\section{Introduction}

Batoid fishes (such as the manta ray) have been object of several studies in the past years, not only in biology [1] [2] [3] but also in the engineering field [4] [5]. The reasons for the interest in these fishes lie in some peculiar aspects they exhibit, such as their unique morphology and locomotion strategy (sometimes identified as a form of underwater flight). Those aspects confer them interesting capabilities, that are desirable also for artificial machines. The smallest species are extremely agile and quick. The biggest ones still preserve agility and high maneuverability (they can, for example, perform turn-on-a-dime maneuvers), in addition to being very efficient swimmers capable of cruising for very long

\footnotetext{
* These authors contributed equally to this work.
} 
distances. In related literature biologists focused on the odd anatomy of these fishes [1] [2], engineers investigated the fluid-dynamics of their propulsion mechanism [4] [3], while robotics studies tried to replicate their compliant morphology and actuation [5]. Despite the large amount of works on this topic, most of contributions embrace an experimental approach, while less efforts were put in modeling the interaction between morphology and environment. In this work we study the adaptability of a batoid-inspired wing to different environments, using a model-based synthetic methodology. The objective is twofold: investigate how different environments can lead to the evolution of different morphologies and behaviors, while offering an additional example of morphological computation and embodied intelligence. Possible applications are novel adaptable under-water soft robots.

The paper is organized as follows. In Sect. 2 we summarize generalities on Batoid fishes, focusing on peculiarities related to their locomotion mechanism. Section 3 illustrates how the fundamental aspects related to our study are synthesized in a mathematical model, with complex features condensed in few, elegant, parameters. Then we describe the genetic optimization set-up used to investigate the co-evolution of morphology and control in order to achieve optimal swimming in different environments (Sect. 4). The paper ends presenting simulation results (Sect. 5) and discussing future perspectives (Sect. 6).

\section{Biological Inspiration}

Batoid fishes (e.g. electric rays, saw-fishes, guitar-fishes, skates and stingrays) are a monophyletic group of over 500 elasmobranch species nested within sharks [4]. They are distinguished by their flattened bodies, enlarged pectoral fins that are fused to the head, and gill slits that are placed on their ventral surfaces [6] [2] [7]. Batoid fishes can cruise at high speed and can perform turn-on-a-dime maneuvering, making them rich source of inspiration for bio-mimetic artificial underwater locomotion (e.g., AUV, ROV).

Most of the investigators found that Batoids propel themselves by moving their enlarged pectoral fins in a flapping motion, combined with an undulatory motion (a traveling wave moving in the downstream direction) [4]. Rosenberger [3] analyzed the kinematics of eight species of ray, each with a different swimming motion. He identified a continuous spectrum of motion ranging from rajiform undulation (where multiple traveling waves pass down the fins and body) to mobuliform oscillation (characterized by a broad flapping of the pectoral fins). The combination of these two mechanisms appear to be a key element in the swimming dynamics and it is directly connected with the animal morphology.

In the next section we illustrate how these peculiarities were synthesized in a mathematical model. 


\section{Model}

This work follows a so called synthetic methodology. In this section we describe how the synthesis was performed, both for what concerns the definition of a batoid-like morphology and the physical modeling of the swimming dynamics.

\subsection{Morphology}

As for morphology, two features being exhibited by batoid fishes are of particular interest for roboticists. a) Compliance: batoids have elastic, flexible, compliant bodies; b) Under-actuation: most of batoid fishes swim using pectoral fin locomotion [3], i.e. they actuate only the frontal edge of their wings. From a robotics stand-point, this means that their body, featuring - theoretically - an infinite number of degrees of freedom (DOFs), is under-actuated. Moreover, the control of pectoral fins appears to be quite simple, consisting just in oscillatory movements. Compliance and under-actuation make batoids interesting case studies also for embodied intelligence and morphological computation: a rich behavior emerges from the strong interaction with the environment, without the need of a complex control. The above mentioned features are highly relevant to our study, being involved in the formation of the oscillatory and undulatory behaviors responsible of thrust production. Those traits were thus synthesized in our model. First of all, modeling a single wing was sufficient for our intended study. Furthermore, in order to simplify the continuous structure without losing the essential features, we discretized the morphology by means of small rigid segments (Fig. 1). We modeled the wing by means of 10 adjacent radial chains, each being composed by a variable number (10 in the longest chain) of rigid prismatic segments $(22 \times 24 \times 5 \mathrm{~mm})$. This discretization was selected as a trade-off between accuracy and computational cost. The resulting fin shape is approximately semi-elliptic, with root chord of $240 \mathrm{~mm}$ and span of $220 \mathrm{~mm}$, following the experimental set-up presented in [4]. The segments are interconnected by means of hinge joints with torsion springs (modeling bending elasticity). Adjacent chains are free to move one respect to the others, with interactions among them being modeled by means of linear springs (Fig. 2). This structure is able to mimic the passive compliance using the minimum number of DOFs. No structural damping was included as it was assumed to be negligible with respect to the one provided by fluid drag forces. Only the joints belonging to the frontal chain are actuated, by means of simple sinusoidal oscillators (the same oscillation is applied at each actuated joint). Although it may seem oversimplified, the resulting global actuation mimics the one observed in batoids, that do not appear to adopt more complex control strategies (traveling waves are not directly actuated, instead they emerge from the interaction with the environment). This modular, under-actuated, structure is able to reproduce both the undulatory (longitudinal waves) and oscillatory (radial waves) modes involved in thrust production, i.e. waving and flapping. 


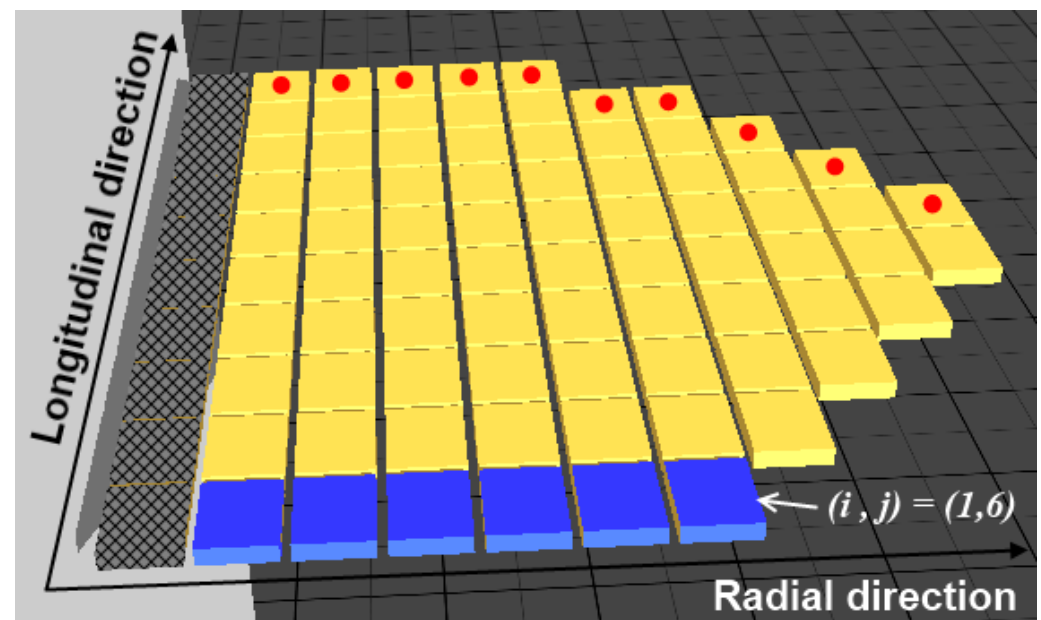

Fig. 1. Discretization of the wing by means of small rigid segments. Segments in the radial direction (index $j$ ) are interconnected by hinge joints: the ones of the first chain are actuated (blue segments), while the others are modeled by passive torsion springs. In the longitudinal direction (index $i$ ) there are no rigid joints: spring forces, better described in Fig. 2, tend to keep adjacent chains on the same plane. Red dots denote the characteristic points of the trailing edge where the amplitude $A$ is sampled. Crossed segments are, instead, fixed.

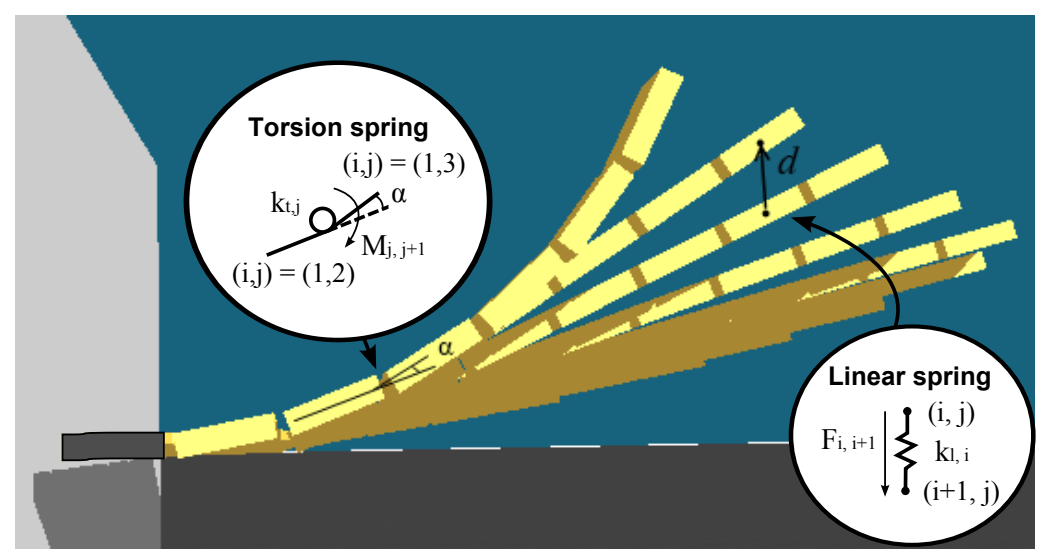

Fig. 2. Displacements and spring forces. The figure shows an angular displacement $(\alpha)$ between two adjacent segments (segment $j$ and $j+1$ respectively) in the radial direction. A torsion spring couple $M_{j, j+1}=k_{t, j} \cdot \alpha$ is applied between the two. $k_{t, j}$ is fixed for all the hinge joints having the same distance from the body. The figure also shows a displacement ( $d$, vector) between the center of mass of two adjacent segments in the longitudinal direction (segments $i$ and $i+1$ ). A force $F_{i, i+1}=k_{l, i} \cdot d$ is applied between the two centers of mass, with $k_{l, i}$ being fixed for all segments at the same distance from the body. 


\subsection{Batoids swimming dynamics}

As for most fishes, swimming locomotion of batoids is dominated by vortices dynamics. Modeling this kind of phenomena can be very hard, however various experimental investigations have been done, showing that these complex fluid phenomena can all be described by means of some elegant non-dimensional parameters. The most important one is arguably the Strouhal number. Firstly introduced in 1915 by Lord Rayleight in order to describe vortex phenomena, it was then widely used in the description of fishes locomotion, and it is defined as $[8][9]$

$$
S t=\frac{f A}{U},
$$

where $U$ is the average swimming speed and $f$ and $A$ are respectively the frequency and peak-to-peak amplitude of the characteristic locomotion swimming movement (e.g. tail beating for fishes, fins flapping for batoids). In [8] different fishes over a large range of Reynolds numbers have been studied, finding that they all tend to cruise (i.e. swim at constant speed) in the same Strouhal number range $(0.25<S t<0.35)$. Authors suggested that this may correspond to the most efficient cruising condition (confirmed by other studies [9]). The other relevant parameters governing the phenomenon are the Reynolds number $(R e)$ and the phase $(\phi)$ of the undulatory waves. In order to describe the phenomenon we built a mathematical model able to describe the dynamics of the batoid fin and its interaction with the fluid, modeled through local drag forces (more details in Sect. 5). The main goal of this model is to show how the interaction between the fluid (mainly described by viscosity $\mu$ and density $\rho$ ) and the compliant, under-actuated, body structure leads to the emergence of different combinations of flapping and waving, as observed among batoid species.

In order to compute the Strouhal number, the amplitude $A$ is estimated as the mean transverse oscillation of the trailing edge, sampled in a number of discrete points (Fig. 1). On the other hand, the frequency $f$ is a parameter, while the mean cruise speed $U$ is assumed to be constant $(U=0.11 \mathrm{~m} / \mathrm{s})$, referring to a similar set-up present in literature [4].

\section{Optimization setup}

The model was implemented in the Webots simulation environment [10]. As for the optimization study, we used genetic algorithms offered by the MATLAB $2011 b$ Global Optimization Toolbox (The MathWorks Inc., Natick, MA, 2000). We set-up the problem as a bounded optimization, adopting a real-coded genetic algorithm based on the Augmented Lagrangian Genetic Algorithm (ALGA) [11]. An adaptive feasible mutation operator and a scattered crossover operator are adopted. The chosen population size is of 50 individuals, the maximum number of generations was set to 1000 , and the termination criterion was the best individual reaching a fitness of 0 . 
Parameters We co-evolved both control and morphological parameters. The first consist of amplitude $(a)$ and frequency $(f)$ of the oscillators driving the active joints. For the latter we assumed both torsion and linear springs to be constant at a given distance from the body. The adopted discretization consists in 10 torsion springs coefficients $\left(k t_{j}\right)$ and 10 linear springs coefficients $\left(k l_{j}\right)$. The 22 evolved parameters are thus:

$$
\left[A, f, k t_{1}, \ldots, k t_{10}, k l_{1}, \ldots, k l_{10}\right]
$$

Target As explained in Sect. 2, we optimized the Strouhal number resulting from a given set of parameters. As target value, we chose $S t^{*}=0.3$. The particular value is motivated by fluid-dynamics studies on fishes [4] [8] [9], showing that most fishes exhibit a Strouhal number close to the above one.

Fitness Given a set of parameters, the fitness is computed as the normalized, squared difference between the Strouhal number achieved for the current morphology and control $(S t)$ and the target Strouhal number $(S t)$, thus:

$$
\text { fitness }=\left(\frac{S t-S t^{*}}{S t^{*}}\right)^{2}
$$

\section{Results and Discussion}

We investigated the role of density and viscosity as they directly affect drag forces that, interacting with the under-actuated body structure, lead to the emergence of different combinations of flapping and waving. Particularly, we evolved our wing in two different fluids, water $\left(\rho=1000 \mathrm{Kg} / \mathrm{m}^{3}, \mu=1.15 \cdot 10^{-3} \mathrm{~Pa} \cdot s\right)$ and Tetrachloroethylene $\left(\rho=1622 \mathrm{Kg} / \mathrm{m}^{3}, \mu=0.89 \cdot 10^{-3} \mathrm{~Pa} \cdot \mathrm{s}\right)$. It has to be noted that our simulations fall in an interval of Reynolds numbers $\left(\left[2 \cdot 10^{4}, 3 \cdot 10^{5}\right]\right.$, turbulent flow) in which the effect of viscosity variation on the drag coefficient is negligible. Thus the adopted local drag forces are: $F_{d r a g}=\frac{1}{2} C_{d} \rho S_{i j} v_{i j}^{2}$, where the terms are respectively the drag coefficient, the fluid density, the exposed surface of the segment $(i, j)$ and its velocity perpendicular to $S_{i j}$. The optimization was performed with two different choices for the parameters bounds. We kindly invite the reader to watch the accompanying videos at:

http://sssa.bioroboticsinstitute.it/papers/FinEvolution

\subsection{Wide bounds}

In a first step, parameters bounds were kept wide in order to let the genetic algorithm explore a larger space of wing configurations. Adopted bounds are reported in Tab. 1. In both the fluid environments the genetic algorithm managed to reach the desired optimal Strouhal number $S t^{*}$, with no residual error (Tab. 2 ). Analyzing $f$ and $a$, it resulted that the wing evolved in water (lower density) 
is actuated at a slightly higher frequency, while the amplitude of the oscillation is slightly smaller. The evolved spring parameters are summarized in Fig. 3. Interestingly, the actuation frequency, the amplitude of emergent waves and their wavelength are consistent with experimental data related to some species of batoids (e.g. Rhinoptera bonasus, Dasyatis violacea [3]), thus supporting the validity of the proposed model. From a qualitative point of view, the waves emerging in the model resemble the ones observed in some reference animals (Fig. 4).

From the achieved results we observed that the problem is under-constrained, meaning that there are several possible solutions that minimize the fitness. In order to reduce the redundancy we decided to shrink the bounds towards more biologically plausible ranges of values. Moreover, the necessity of imposing the continuity of spring parameters emerged to avoid discontinuous behaviors among parameters.

Table 1

Wide parameters bounds.

\begin{tabular}{l|l|l|l|l} 
& $f[\mathrm{~Hz}]$ & $a[\mathrm{rad}]$ & $k l_{j}[\mathrm{~N} / \mathrm{m}]$ & $k t_{j}[\mathrm{~N} / \mathrm{rad}]$ \\
\hline \hline Lower & 0.2 & 0.0349 & 0.05 & 0.005 \\
\hline Upper & 6.0 & 0.349 & 100.0 & 5.0 \\
\hline
\end{tabular}
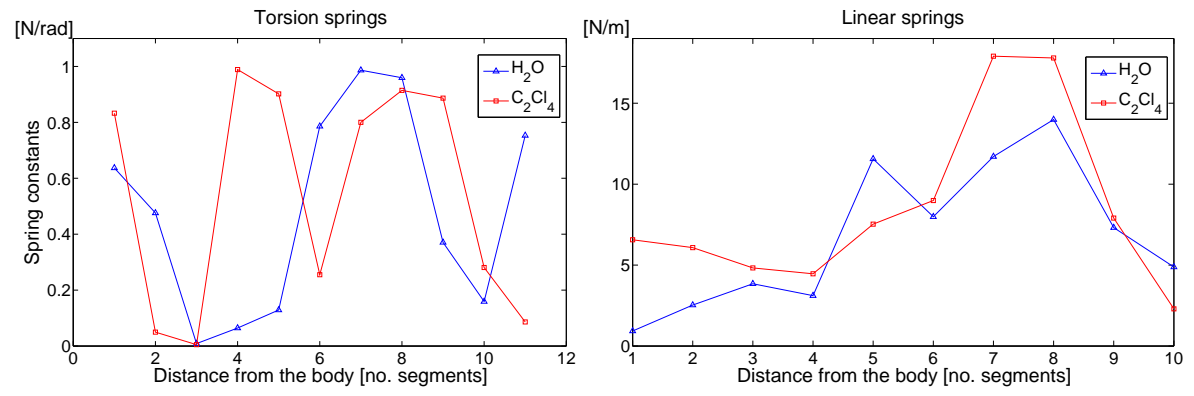

Fig. 3. Evolved spring parameters with wide bounds. Left: torsion springs. The plot highlights that without imposing the continuity of spring parameters results can be irregular, not biologically plausible, and difficult to interpret. Right: linear springs. The wing evolved in the tetrachloroethylene is more rigid (almost everywhere) than the one evolved in water. In this case resulting spring parameters are reasonably smooth. 

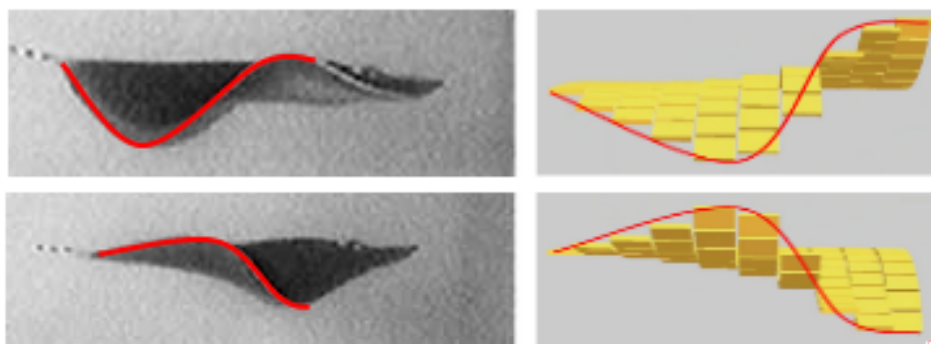

Fig. 4. Comparison of wing profiles in two different instants. Left: Gymnura micrura (adapted from [3]). Right: The proposed model, evolved for water environment.

Table 2

Optimization results. Actuation frequency and amplitude, mean emergent amplitude on the trailing edge, Strouhal number are reported.

\begin{tabular}{l|l|l|l|l|l} 
& Fluid & $\begin{array}{l}f \\
{[\mathrm{~Hz}]}\end{array}$ & $\begin{array}{l}a \\
{[\mathrm{rad}]}\end{array}$ & $\begin{array}{l}A \\
{[\mathrm{~m}]}\end{array}$ & St \\
\hline \hline \multirow{2}{*}{ Wide bounds } & Water & 0.944 & 0.232 & 0.035 & 0.300 \\
\cline { 2 - 6 } & Tetra chloro ethylene & 0.887 & 0.245 & 0.037 & 0.300 \\
\hline \multirow{2}{*}{ Narrow bounds } & Water & 0.737 & 0.261 & 0.0448 & 0.300 \\
\cline { 2 - 6 } & Tetra chloro ethylene & 1.06 & 0.262 & 0.0311 & 0.300 \\
\hline
\end{tabular}

\subsection{Narrow bounds}

A first refinement consists in imposing the continuity of spring parameters. In order to impose such constraint, we assume the thickness of the fin to vary linearly on the radial direction. It is thus reasonable to model the variation of spring parameters with a cubic polynomial, given that the bending elasticity is a cubic function of the thickness. The resulting non-dimensional equations are:

$$
\begin{gathered}
\widetilde{k l_{j}}=1+a l_{3} \cdot{\widetilde{x_{j}}}^{3}+a l_{2} \cdot{\widetilde{x_{j}}}^{2}+a l_{1} \cdot \widetilde{x_{j}} \\
\widetilde{k t_{j}}=1+a t_{3} \cdot \widetilde{x_{j}}{ }^{3}+a t_{2} \cdot{\widetilde{x_{j}}}^{2}+a t_{1} \cdot \widetilde{x_{j}},
\end{gathered}
$$

where:

$$
\begin{array}{ll}
\widetilde{k l_{j}}=k l_{j} / k l_{\max }, & x_{j}=j \cdot d x, \\
\widetilde{k t_{j}}=k t_{j} / k t_{\max }, & d x=22 \mathrm{~mm}, \\
\widetilde{x_{j}}=x_{j} / x_{\max }, & j=0, \ldots, 9 .
\end{array}
$$

Consequently, the optimization parameters are reduced to 10, being:

$$
\left[f, a, a l_{3}, a l_{2}, a l_{1}, k l_{\max }, a t_{3}, a t_{2}, a t_{1}, k t_{\max }\right] .
$$


The non-dimensional cubic polynomials were built in order to span from a constant behavior $\left(k_{j}=k_{\max }\right)$ to a full cubic $\left(a_{3}=a_{2}=a_{1}=-1\right)$ going to zero at the tip of the fin, allowing all the possible combinations between these boundaries (Fig. 5). The resulting bounds for the polynomial parameters range from -1 to 0 . It has to be noted that among the resulting curves, only those that are positive for all the sampling points are admissible. This was imposed applying a constraint in the genetic algorithm. Limiting the bounds for the $a_{i}$ coefficients to avoid negative curves could have discarded meaningful solutions.

Narrowing the bounds is motivated by the need to reduce the redundancy of the problem as well as to define a more physically plausible parameters range. Bounds related to actuation frequency $(f)$ and amplitude $(a)$ are consistent with the range of behaviors observed in batoid fishes [3]. As for $k l_{\max }$ and $k t_{\max }$, the relative bounds were defined by testing several limit conditions for the parameters, and observing the resulting behavior of the wing (Fig. 6). Parameters bounds are summarized in Tab. 3.

Results of the genetic optimization are summarized in Tab. 2. Resulting actuation amplitude $(a)$ does not vary between the two fluids, while there is a significant difference in the frequency $(f)$. Particularly, the actuation frequency results to be higher for the wing evolved in Tetrachloroethylene, showing an inverse trend respect to the simulation featuring wide bounds. This discrepancy arises from the under-constrained nature of the problem: it is thus possible to achieve more than one result exhibiting the optimal fitness. Given the more biologically plausible set-up, results achieved in presence of continuous spring constants and narrow bounds are arguably more reliable.

As for torsion springs (Fig. 7), the behavior is almost identical in the two fluid environments, with a steep variation along the span of the fin. Pronounced differences can be instead observed in linear springs coefficients (Fig. 7). The wing evolved in water is softer, and exhibits more variation in the radial direction. As a result, a greater presence of undulation respect to oscillation is observed for the wing evolved in the Tetrachloroethylene.

Table 3

Narrow bounds parameters.

\begin{tabular}{l|l|l|l|l|l|l} 
& $\begin{array}{l}f \\
{[\mathrm{~Hz}]}\end{array}$ & $\begin{array}{l}a \\
{[\mathrm{rad}]}\end{array}$ & $a l_{i}$ & $\begin{array}{l}k l_{\max } \\
{[\mathrm{N} / \mathrm{m}]}\end{array}$ & $a t_{i}$ & $\begin{array}{l}k t_{\max } \\
{[\mathrm{N} / \mathrm{rad}]}\end{array}$ \\
\hline \hline Lower & 0.5 & 0.0873 & -1.0 & 1.0 & -1.0 & 0.01 \\
\hline Upper & 5.0 & 0.2618 & 0.0 & 10.0 & 0.0 & 0.1 \\
\hline
\end{tabular}




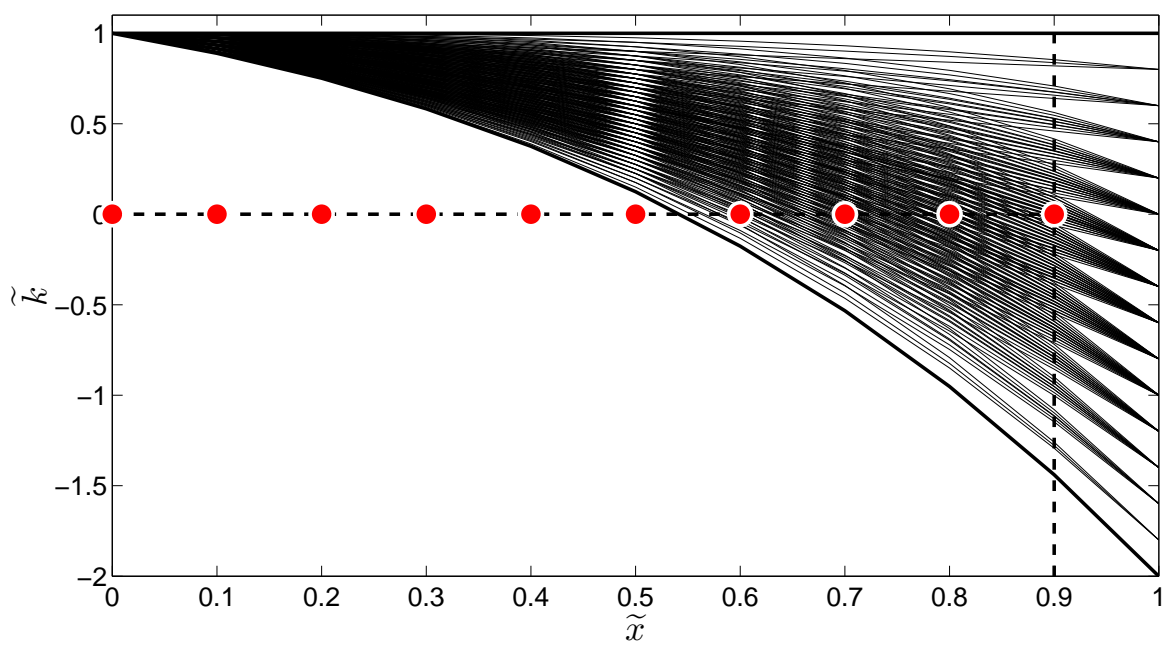

Fig. 5. Non-dimensional cubic polynomials used to model spring constants, plotted with a parameters step of 0.2. Limit curves are thicker. Red dots denote the sampling points $x_{j}$. Only the curves that are positive for all the sampling points are admissible.
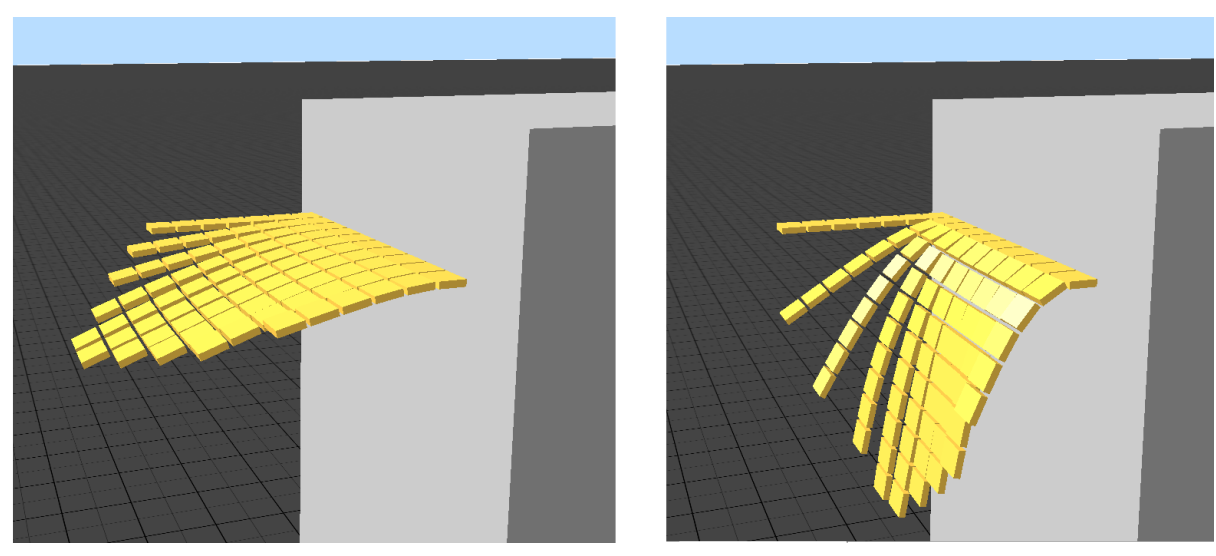

Fig. 6. Free fall of the fin in air when the parameters (linear and torsion springs) are set to the upper and lower bounds (left and right, respectively). In these tests the position of the frontal chain is kept fixed, to sustain the fin. 

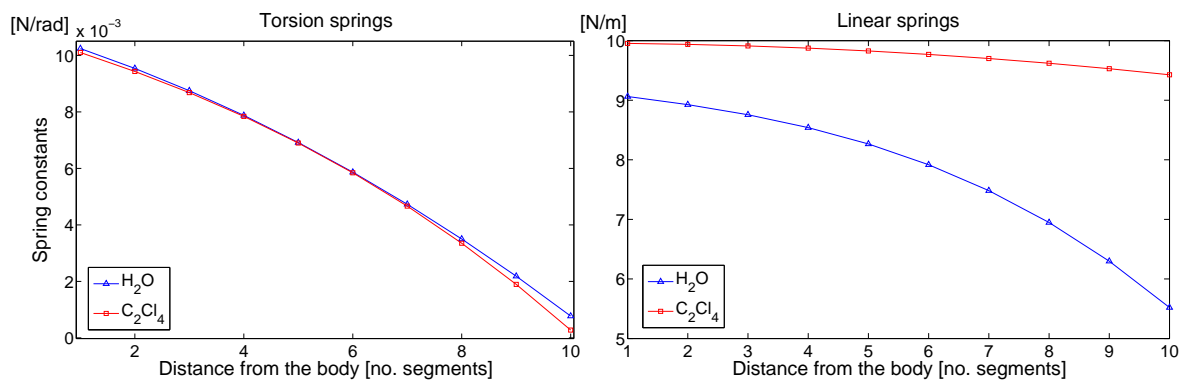

Fig. 7. Evolved spring parameters with narrow bounds. Left: torsion springs. The behavior is almost the same in the two fluid environments, with a steep variation along the span of the fin. Right: linear springs. In this case a pronounced difference can be seen between the two environments, with the wing evolved in water being softer and exhibiting more variation in the radial direction.

\section{Conclusions}

A model for an under-actuated, compliant wing was presented and optimized by means of genetic algorithms for different fluid properties. The optimization target was motivated by fluid-dynamics studies conducted on fishes, showing a correlation between Strouhal number and swimming efficiency. Simulation results show a good convergence of the genetic algorithm, able to find sets of morphological and actuation parameters which perfectly match the desired Strouhal number in our model. Moreover, the main characteristic quantities are consistent with biological data, thus supporting the validity of the proposed model.

Present results encourage the investigation on speciation of batoid fishes in different environments by means of modeling tools. This will require to enrich the fluid dynamics set-up (e.g. modeling the relationship between emergent oscillations and thrust) as well as the capability of defining morphologies able to span the variety of batoid shapes. From a robotics perspective, the long-term vision is that of a bio-mimetic robot able to adapt to different fluids and, possibly, to different flow conditions, adjusting both morphology and actuation in order to exploit the interaction with the environment letting an efficient, effective swimming behavior emerge. Besides contributing to the fields of embodied intelligence and morphological computation, this could foster practical soft-robotics applications such as underwater robots for environmental monitoring and inspection of industrial plants.

\section{Acknowledgments}

This work is supported by RoboSoft - A Coordination Action for Soft Robotics (FP7-ICT-2013-C \# 619319). We would also like to acknowledge the ShanghAI Lectures project and the teaching assistants for the inspiration and assistance. 


\section{References}

1. Schaefer, J.T., Summers, A.P.: Batoid wing skeletal structure: novel morphologies, mechanical implications, and phylogenetic patterns. Journal of Morphology 264(3) (2005) 298-313

2. Aschliman, N.C., Claeson, K.M., McEachran, J.D.: Phylogeny of batoidea. Biology of sharks and their relatives. Edited by JC Carrier, JA Musick, and MR Heithaus. Indiana University Press, Bloomington, Ind (2012) 57-94

3. Rosenberger, L.J.: Pectoral fin locomotion in batoid fishes: undulation versus oscillation. Journal of Experimental Biology 204(2) (2001) 379-394

4. Clark, R., Smits, A.: Thrust production and wake structure of a batoid-inspired oscillating fin. Journal of fluid mechanics 562(1) (2006) 415-429

5. Suzumori, K., Endo, S., Kanda, T., Kato, N., Suzuki, H.: A bending pneumatic rubber actuator realizing soft-bodied manta swimming robot. In: Robotics and Automation, 2007 IEEE International Conference on, IEEE (2007) 4975-4980

6. Nelson, J.S.: Fishes of the World. John Wiley \& Sons (2006)

7. Dunn, K.A., Miyake, T.: Interrelationships of the batoid fishes (chondrichthyes: Batoidea). Interrelationships of fishes (1996) 63

8. Triantafyllou, G., Triantafyllou, M., Grosenbaugh, M.: Optimal thrust development in oscillating foils with application to fish propulsion. Journal of Fluids and Structures 7(2) (1993) 205-224

9. Eloy, C.: Optimal strouhal number for swimming animals. Journal of Fluids and Structures 30 (2012) 205-218

10. Michel, O.: Webots: Professional mobile robot simulation. Journal of Advanced Robotics Systems 1(1) (2004) 39-42

11. Conn, A.R., Gould, N.I., Toint, P.: A globally convergent augmented lagrangian algorithm for optimization with general constraints and simple bounds. SIAM Journal on Numerical Analysis 28(2) (1991) 545-572 